\title{
Decidualização Ectópica: Uma Entidade Esquecida
}

\section{Ectopic Decidualization: A Forgotten Entity}

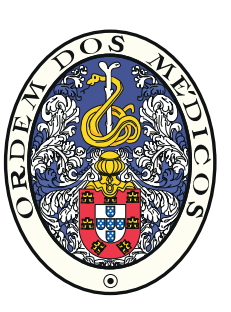

Joana MENDES $\triangle^{1}$, Antónia COSTA 2,3

Acta Med Port 2016 Jan;29(1):63-72

RESUMO

Introdução: Apesar da decidualização ectópica ser uma entidade frequentemente subdiagnosticada, pode ter impacto clínico adverso na morbimortalidade materno-fetal. O objetivo deste trabalho foi rever a evidência científica relativa a etiopatogenia, clínica, abordagem diagnóstica e terapêutica sobre esta temática.

Material e Métodos: A pesquisa bibliográfica foi realizada na PubMed, Web of Science e Scopus, através da query ('deciduosis' OR 'ectopic decidualization' OR 'ectopic decidua' OR 'ectopic decidua reaction'), incluindo-se artigos publicados até 31/6/2014 e de todos os níveis de evidência.

Resultados: A decidualização ectópica, geralmente, representa uma condição benigna, assintomática e sem necessidade de intervenção terapêutica. Encontra-se, maioritariamente, associada à gravidez, com regressão completa no período pós-parto. A frequência do seu diagnóstico depende da suspeição clínica, bem como do local onde surge, sendo o omento e o ovário os locais mais comuns. Quando sintomática, as principais manifestações clínicas são quadros hemorrágicos, nomeadamente hemorragia genital e hemoperitoneu. Os diagnósticos diferenciais incluem patologia maligna, sendo essencial, nestas situações, a confirmação histopatológica. O baixo índice de suspeição clínica pode levar à realização de biópsia, que pode acarretar impacto adverso grave devido à elevada friabilidade destas lesões.

Discussão e Conclusão: O reconhecimento desta entidade e das suas características clínicas torna-se essencial na conduta destas doentes. Tal permite por um lado a abordagem médica precoce e adequada nos casos graves, e por outro lado (na maioria dos casos) manter a atitude expectante minimizando a iatrogenia, mantendo o desfecho favorável da decidualização ectópica.

Palavras-chave: Complicações na Gravidez; Decídua; Gravidez.

\section{ABSTRACT}

Introduction: Although ectopic decidualization is an entity often underdiagnosed, it may have adverse clinical impact on maternal-fetal morbimortality. The objective of this study was to review the scientific evidence regarding the etiology, clinical features, diagnosis and therapeutic approach on this issue.

Material and Methods: The search for this literature review was conducted in PubMed, Web of Science and Scopus, through the query ('deciduosis' OR 'ectopic decidualization' OR 'ectopic decidua' OR 'ectopic decidua reaction'), considering articles of all evidence levels published up to 31/06/2014.

Results: Ectopic decidualization is, usually, a benign condition, asymptomatic and does not require therapeutic intervention. It occurs mostly during pregnancy, with complete regression in the postpartum period. The frequency of the diagnosis depends on the clinical suspicion and its location, being the omentum and the ovary the most common sites. When symptomatic, the main clinical manifestations are genital bleeding and hemoperitoneum. Differential diagnosis includes malignant disease and histopathological confirmation is essential in these situations. The low index of suspicion may lead to a biopsy, which can have serious adverse outcomes due to the high friability of these lesions.

Discussion and Conclusion: The recognition of this entity and its clinical features are essential for the management of these patients. On one side this allows an early and proper medical approach in severe cases, on the other side (the majority of cases) maintaining an expectant attitude avoiding iatrogeny, does not compromise, in most cases, the favorable outcome of ectopic decidualization.

Keywords: Decidua; Pregnancy; Pregnancy Complications.

\section{INTRODUÇÃO}

A célula decidual é uma célula mesenquimatosa embrionária, derivada do tecido conjuntivo laxo da mucosa uterina. $^{1}$

A transformação decidual destas células da mucosa uterina ocorre durante a gravidez e, normalmente, abrange a mucosa do corpo uterino, poupando o colo e o istmo. Denomina-se decidualização ectópica (DE), quando este processo ocorre em qualquer outra localização fora do endométrio. $^{2}$

Neste contexto, podem-se considerar três conceitos distintos $^{3}$ : (1) pré-decídua (reação celular do endométrio, que se forma entre o $23^{\circ}$ e o $28^{\circ}$ dia do ciclo menstrual normal e é citologicamente indistinguível da verdadeira decídua), (2) decídua e (3) DE ou deciduose. Esta última pode ocorrer, com decrescente frequência, em gestações evolutivas in utero, perturbações da gravidez inicial (gravidez ectópica, gestação tópica não evolutiva e doença neoplásica do trofoblasto), e em não grávidas.

É debatido se a deciduose é um fenómeno fisiológico ou patológico. Na literatura existem vários casos clínicos descritos com desfecho adverso quer para a grávida e feto, quer para a mulher não grávida. ${ }^{4-9}$

1. Faculdade de Medicina. Universidade do Porto. Porto. Portugal.

2. Serviço de Ginecologia e Obstetrícia. Centro Hospitalar São João. Porto. Portugal.

3. Departamento de Ginecologia e Obstetrícia. Faculdade de Medicina. Universidade do Porto. Porto. Portugal.

$\triangle$ Autor correspondente: Joana Mendes. joanaritamendes@gmail.com

Recebido: 31 de março de 2015 - Aceite: 03 de agosto de 2015 | Copyright @ Ordem dos Médicos 2016 
A grande variabilidade da sua apresentação clínica faz com que seja um diagnóstico difícil. Contudo, esta entidade deve estar incluída nos diagnósticos diferenciais de avaliações médico-cirúrgicas, imagiológicas e anatomopatológicas.

O presente trabalho tem como objetivo efetuar uma revisão da evidência científica sobre a DE, no que respeita à sua fisiopatologia, abordagem diagnóstica e terapêutica, permitindo alertar para a necessidade do elevado índice de suspeição clínica desta entidade. Sendo a DE frequentemente subdiagnosticada pretende-se otimizar a conduta clínica nestas doentes e, deste modo, minimizar possíveis desfechos adversos.

\section{MATERIAL E MÉTODOS}

Realizámos uma revisão com base na pesquisa bibliográfica eletrónica nas bases de dados bibliográficas PubMed, Web of Science e Scopus, com a seguinte query: 'deciduosis'[All Fields] OR 'ectopic decidualization'[All Fields] OR 'ectopic decidua'[All Fields] OR 'ectopic decidual reaction'[All Fields]. Incluímos todos os artigos publicados até 31 de Junho de 2014, independentemente do nível de evidência. ${ }^{10}$

Os critérios de exclusão foram artigos em duplicados das diferentes bases de dados consultadas, escritos em língua diferente do inglês, francês e português, artigos não adequados ao tema ou sem texto integral disponível. Efetuamos também pesquisa manual a partir das referências bibliográficas das publicações obtidas na pesquisa inicial.

\section{RESULTADOS}

Para a elaboração do presente trabalho obtivemos um total de 74 artigos, dos quais nenhum era de nível de evidência 1 e 2, apenas um artigo com nível de evidência três correspondendo a uma revisão sistemática, ${ }^{11}$ sendo os restantes de nível de evidência quatro, na sua grande maioria descrição de casos clínicos. ${ }^{10}$ (Fig. 1, Tabela 1)

\section{Enquadramento histórico}

A primeira referência à $D E$ é partilhada entre Bayer em 1885 e Walker em 1887 com o achado de células decíduais ao nível do colo uterino durante a gravidez e no peritoneu pélvico num caso de gravidez extrauterina, respetivamente. Mais tarde, em 1905, Hirschberg fez uma revisão do tema e descreveu pela primeira vez um caso de gravidez tubária com deciduose no apêndice, mimetizando granulomas ou neoplasia. Depois destas, outras localizações da deciduose foram descritas, nomeadamente por Taussing, em 1906, que listou diferentes localizações de deciduose num caso de gravidez tubária (mucosa cervical, trompa não grávida, serosa uterina, peritoneu pélvico, ovários, serosa de intestino delgado, omento, apêndice, adenomioma do útero, aderências peritoneais) e por Geipel que, em 1917, acrescentou gânglios linfáticos pélvicos à lista de localizações conhecidas. Nova revisão do tema foi feita por Rosenberger, em 1921, onde foi sugerido que esta reação decidual era secundária a endometriose. Mais tarde, em 1927,
Geipel volta a reportar deciduose desta vez no diafragma e na cápsula esplénica. Todos os casos até então descritos estavam relacionados com gravidez, até Schiller, em 1924, e Schereschewsky, em 1931, a descreverem na ausência de gravidez, não conseguindo determinar se era uma nova forma de manifestação (como decidua mestrualis) ou se a gravidez não tinha sido diagnosticada. ${ }^{12}$ Wallart, em 1936, relata sete casos de DE no ovário em amostras de necrópsia de mulheres pós-menopausa. ${ }^{13} \mathrm{Em} \mathrm{1940,} \mathrm{Reis} \mathrm{e}$ Sinykin descrevem um caso de apendicite em grávida com descrição microscópica de deciduose ${ }^{14}$ e, em 1949, Lapan relata três casos de decídua ectópica no colo e vagina, com diagnóstico inicial de malignidade. ${ }^{3}$

\section{Fisiopatologia}

$\mathrm{Na}$ grande maioria dos casos, a deciduose ocorre na gravidez, mas foi também descrita em mulheres não grávidas. A fisiopatologia destas lesões ainda não está totalmente esclarecida, podendo discutir-se vários mecanismos para a formação de tecido decidual extra-uterino:

\section{- Decidualização de lesões endometriais pré-existen- tes, durante a gravidez}

Esta hipótese baseia-se no facto de existir partilha geográfica entre a endometriose e a deciduose, durante a gravidez. ${ }^{15,16}$ Para os seus defensores, a possível inexistência de células glandulares, em amostras histológicas, pode dever-se à sua regressão como consequência do excesso de proliferação de estroma, não sendo argumento para eliminar a hipótese de origem em lesões endometrioides prévias. ${ }^{16,17}$

Histologicamente, a distribuição difusa de lesões peritoneais, edema do estroma decidualizado, focos hemorrágicos antigos e recentes, células 'pseudoxantomatosas' e fibrose traduzem atrofia das glândulas do endométrio que, juntamente com a presença de reação de Arias-Stella, são importantes no diagnóstico da transformação decidual de focos de endometriose na gravidez, isto é, endometriose decidualizada. ${ }^{18}$

Contudo, apesar da deciduose poder estar relacionada com endometriose, certos casos não têm mostrado a consistência dessa associação, pois a lesão regride por completo 4 - 6 semanas após a gravidez. ${ }^{19-22}$ Por outro lado a progesterona parecer induzir a DE (ao invés dos progestativos utilizados para suprimir a endometriose), favorecendo a hipótese de se tratar de duas entidades distintas. ${ }^{23}$

\section{- Metaplasia decídual}

Esta teoria, que surgiu em consequência das lacunas da teoria anterior, prevê o desenvolvimento da decídua ectópica a partir da metaplasia de células estromais pluripotenciais do mesênquima subcelómico, sob influência de fatores hormonais. ${ }^{11,17,19,24-26}$

Existe uma área submesotelial, de difícil visualização microscópica, que pode originar diferentes tipos celulares: fibroblastos, miofibroblastos e leiomiócitos. ${ }^{20}$ O estrogénio induz a proliferação de fibroblastos e, quando este se 


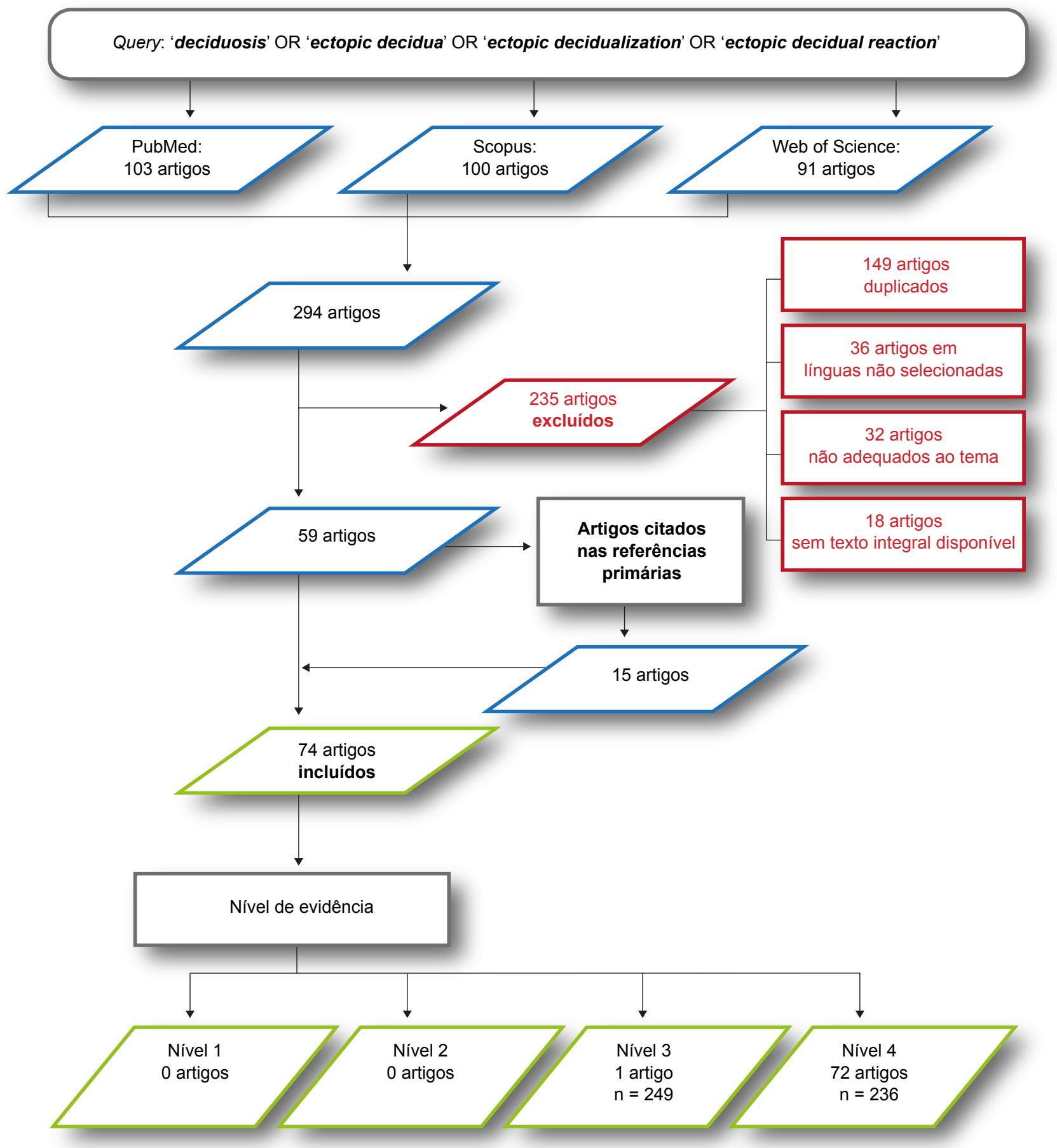

Figura 1 - Resultados da pesquisa bibliográfica

n: número total de casos descritos

encontra associado à progesterona, favorece a sua diferenciação em leiomiócitos e células decíduais. ${ }^{11}$

Abramowicz et $\mathrm{al}^{27}$ afirmam que a DE parece localizar-se preferencialmente ao nível de estruturas tecidulares derivadas do epitélio celómico: omento e peritoneu. Estas estruturas apresentam uma origem embrionária comum com os canais de Müller que dão origem ao útero e à parte superior da vagina.

Zaytsev et al $^{19}$ relataram os casos de 10 grávidas com diagnóstico histológico de DE comprovada de amostras de tecido submesotelial peritoneal obtidas no decurso de intervenções cirúrgicas (nove no decurso de cesarianas e uma no decurso de apendicectomia), de diferentes locais dentro da cavidade abdominal. Nenhum dos casos tinha história de endometriose prévia.

Buttner et al, ${ }^{20}$ questionados se a DE é uma transformação estromal esporádica ou uma reação normal do estroma peritoneal extra-uterino durante a gravidez que desaparece no pós-parto, demonstraram o processo de decidualização de fibroblastos durante uma análise sistemática citológica 
Tabela 1 - Sumário de casos clínicos descritos na literatura de decidualização ectópica durante e na ausência gravidez

\begin{tabular}{|c|c|c|}
\hline Autor, ano & Localização da decidualização ectópica & $\mathbf{n}$ \\
\hline \multicolumn{3}{|l|}{ Gravidez } \\
\hline Taussing, $1906^{1}$ & Colo uterino, trompa de Falópio, peritoneu, omento, apêndice & 1 \\
\hline Reis, $1940^{14}$ & Apêndice & 1 \\
\hline Sanes, $1943^{49}$ & Apêndice & 2 \\
\hline Bettinger, $1947^{64}$ & Rim & 1 \\
\hline Lapan, $1949^{3}$ & Colo uterino, vagina & 3 \\
\hline Melody, $1950^{29}$ & Omento & 1 \\
\hline Israel, $1954^{36}$ & Ovário & 21 \\
\hline McGee, $1955^{24}$ & Colo uterino & 27 \\
\hline Lepage, $1955^{2}$ & Colo uterino & 15 \\
\hline Bret, $1959^{70}$ & Colo uterino & 14 \\
\hline Orr, $1961^{15}$ & Parede anterior da vagina & 1 \\
\hline Le Coz, $1967^{25}$ & Colo uterino & 8 \\
\hline Hulme-Moir, $1969^{30}$ & Apêndice, omento & 1 \\
\hline Covell, $1977^{53}$ & Gânglios pélvicos & 1 \\
\hline Herr , $1978^{37}$ & Ovário & 21 \\
\hline Chapman, $1979^{43}$ & Colo uterino & 1 \\
\hline Schneider, $1981^{42}$ & Colo uterino & 59 \\
\hline Mills, $1983^{54}$ & Gânglios para-aórticos & 1 \\
\hline Richter, $1983^{48}$ & Peritoneu & 1 \\
\hline Burnett , $1986^{28}$ & Gânglio pélvico & 1 \\
\hline Zaytsev, $1987^{19}$ & Gânglio para-aórtico, peritoneu, apêndice, omento & 10 \\
\hline Cobb, $1988^{16}$ & Gânglio pélvico & 1 \\
\hline Gilardi, $1988^{66}$ & Colo uterino & 20 \\
\hline Suster, $1990^{50}$ & Apêndice & 6 \\
\hline Courtin, $1991^{58}$ & Pulmão & 1 \\
\hline Buttner, $1993^{20}$ & Omento & 60 \\
\hline Bashir, $1995^{55}$ & Íleo terminal, cego, cólon ascendente & 1 \\
\hline Mizumoto, $1996^{56}$ & Parede anterior do útero & 1 \\
\hline Flieder, $1998^{59}$ & Pulmão & 1 \\
\hline Kularbkaew, $1998^{57}$ & Face posterior do útero & 1 \\
\hline Fair, $2000^{61}$ & Nódulos cutâneos: vulva e umbigo & 2 \\
\hline Gornall, $2000^{21}$ & Colo uterino & 1 \\
\hline Malpica, $2002^{38}$ & Peritoneu, ovário & 1 \\
\hline Aziz, $2004^{9}$ & Peritoneu & 1 \\
\hline Kondi-Pafiti, $2005^{31}$ & Omento & 3 \\
\hline Wu, $2005^{17}$ & Gânglio pélvico & 1 \\
\hline O'Leary, $2006^{5}$ & Ovários, colo uterino & 1 \\
\hline Rodriguez, $2006^{51}$ & Íleo terminal, cego, apêndice & 1 \\
\hline Kinra, $2006^{39}$ & Peritoneu, ovário, cólon sigmóide, omento & 1 \\
\hline Machida, $2008^{40}$ & Ovário & 3 \\
\hline
\end{tabular}


Tabela 1 - Sumário de casos clínicos descritos na literatura de decidualização ectópica durante e na ausência gravidez

\begin{tabular}{|c|c|c|}
\hline Autor, ano & Localização da decidualização ectópica & $\mathbf{n}$ \\
\hline \multicolumn{3}{|l|}{ Gravidez } \\
\hline Shukla, $2008^{32}$ & Omento, trompas de Falópio & 3 \\
\hline Dharan, $2009^{8}$ & Gânglio pélvico & 1 \\
\hline Ellis, $2009^{23}$ & Peritoneu & 1 \\
\hline Lesaffer, $2009^{52}$ & Apêndice & 2 \\
\hline Szopinski, $2009^{65}$ & Parede posterior da bexiga & 1 \\
\hline Kim, $2010^{60}$ & Pulmão, diafragma & 1 \\
\hline Acuda, $2011^{33}$ & Ovário, omento & 1 \\
\hline Park, $2011^{7}$ & Hemicólon, ovário, trompa de Falópio & 1 \\
\hline Bolat, $2012^{22}$ & Peritoneu & 7 \\
\hline Natale, $2012^{62}$ & Nódulos cutâneos: períneo e região supra-púbica & 2 \\
\hline Kondoh, $2012^{34}$ & Peritoneu, omento & 1 \\
\hline Boztosun, $2012^{41}$ & Ovário & 1 \\
\hline Dogan, $2012^{44}$ & Peritoneu, apêndice & 1 \\
\hline Adhikari, $2013^{45}$ & Peritoneu & 1 \\
\hline Galao, $2013^{35}$ & Omento & 1 \\
\hline Nishikawa, $2013^{6}$ & Intestino & 8 \\
\hline Semenova, $2013^{26}$ & Parede anterior e posterior do útero & 2 \\
\hline Abramowicz, 2014² & Peritoneu, omento & 1 \\
\hline Cruz, $2014^{47}$ & Peritoneu & 1 \\
\hline Salehgargari, $2014^{46}$ & Peritoneu & 1 \\
\hline \multicolumn{3}{|l|}{ Ausência de gravidez } \\
\hline Ober $1957^{13}$ & Ovário & 16 \\
\hline Tang $1985^{4}$ & Trompa de Falópio & 1 \\
\hline
\end{tabular}

n: número de casos

e imunológica de 60 biópsias do grande omento de cesarianas e dois casos de gravidezes tubárias submetidas a intervenção cirúrgica. Na microscopia de fragmentos do grande omento, $97 \%$ dos casos apresentavam lesões de decídua ectópica focais e $3 \%$ lesões difusas. Estes achados demonstram a existência de uma metaplasia fisiológica do mesênquima submesotelial, induzido por influência hormonal durante a gravidez, que ocorre em quase todos as gestações após o primeiro trimestre.

A transformação decidual, tanto no endométrio como no ovário, foi observada também com lesões proliferativas de trofoblasto como, por exemplo, em coriocarcinoma. ${ }^{13}$ A incapacidade de observar as reações decíduais sob as superfícies peritoneais em homens com coriocarcinoma, genital ou extra-genital, é evidência adicional de que a progesterona elaborada pela célula luteínica é um elo necessário na cadeia. ${ }^{13}$

O desenvolvimento de decídua na ausência de gravidez, isto é, sem trofoblasto, presume que a transformação decidual não seja o resultado do estímulo direto da gonadotrofina coriónica. Tal transformação em mulheres não grávidas ou pós-menopausa pode ser explicada pela es- timulação progestativa derivada de um corpo lúteo ativo, hormonas progesterona-like do córtex da supra-renal ${ }^{13,20}$ ou contraceção progestativa injetável de longa duração. ${ }^{4}$ A presença de doença trofoblástica ou resposta a trauma local/irradiação da pelve em estroma sensível podem também ser fatores associados à deciduose não relacionada com gestação. ${ }^{13}$

\section{- Disseminação linfática de células benignas com ori- gem no endométrio}

A hipótese da deciduose poder resultar da disseminação linfática é defendida pela evidência da presença de depósitos decíduais em gânglios linfáticos, por vezes denominada 'metástase benigna'. ${ }^{17,28}$

\section{Outras teorias}

A presença de lesões inflamatórias, trauma local ou irradiação pélvica foram também descritas como fatores de predisposição para o desenvolvimento de deciduose. ${ }^{13,15}$ Le Coz et al ${ }^{25}$ descreve a teoria da implantação, em que traumatismos anteriores tais como curetagem, eletrocoagulação ou parto distócico são promotores de 
deciduose cervical. As células do mesênquima subcelómico serão libertadas e depositam-se no trato genital inferior.

\section{Epidemiologia}

A frequência com que o diagnóstico de DE é efetuado depende, em certa medida, do local onde possa surgir, do índice de suspeição, dos critérios de diagnóstico, bem como do tipo de abordagem diagnóstica, nomeadamente a forma como é realizada e os critérios de realização de biopsia.

A transformação decidual ectópica associada à gravidez foi descrita em vários locais: omento ${ }^{19,20,27,29-35}$ (em $100 \%$ dos casos), ${ }^{20}$ ovário $^{5,7,13,33,36-41}$ (em 90,5\% - 100\% dos casos), ${ }^{36,37}$ colo uterino ${ }^{2,3,5,21,24,25,36-38}$ (em 10\% - 36,5\% dos casos), ${ }^{15,19,24,25,42,43}$ trompa de Falópio ${ }^{4,7,19,32}$ (em 5,5\% dos casos), ${ }^{19}$ peritoneu, ${ }^{9,22,23,27,34,38,39,44-48}$ apêndice, ${ }^{14,19,30,44,49-52}$ gânglios linfáticos, $8,16,17,19,28,53,54$ intestino, ${ }^{6,7,39,51,55}$ serosa uterina, ${ }^{26,56,57}$ vagina, ${ }^{3,15}$ pulmão, ${ }^{58-60}$ pele,${ }^{61,62}$ miométrio, ${ }^{63}$ diafragma, ${ }^{60}$ rim $^{64}$ e bexiga. ${ }^{65}$

O caso de deciduose mais precocemente descrito foi à sexta semana de amenorreia numa gravidez ectópica, mas, regra geral, ocorre durante o segundo e terceiro trimestres de gestação. ${ }^{19}$ Está descrito o seu início entre a $8^{a}$ e $16^{a}$ semana de gestação, quando falamos de deciduose cervical, ${ }^{66}$ ou depois da nona semana, em casos de deciduose ovárica; ${ }^{67}$ outros ainda relatam o seu surgimento sobretudo no terceiro trimestre. ${ }^{33}$

Esta entidade desaparece rapidamente no período pós-parto, quatro a seis semanas de puerpério. ${ }^{20,22,39,43}$

\section{Clínica}

A DE é habitualmente um processo assintomático, de evolução benigna e geralmente subclínico, sendo um achado incidental em contexto cirúrgico (cesariana, cirurgia em contexto de gravidez ectópica, laqueação tubária pós-parto, apendicectomia, etc.). As suas manifestações clínicas dependem sobretudo da sua localização e extensão. ${ }^{22,26,33}$ (Tabela 2)

Os principais sintomas relatados na literatura são os hemorrágicos, que vão desde a perda hemática genital, ${ }^{2,3,15,16,24,25}$ hemoperitoneu (durante a gravidez, ${ }^{6,9,26,29,34,55,56}$ intraparto ${ }^{7}$ e pós-parto ${ }^{5,7,30,41,48}$ ou em casos não associados a gravidez), ${ }^{4}$ até hemorragia digestiva baixa; ${ }^{6,55}$ podendo estar associada a risco de vida materna e/ou morte fetal. ${ }^{9,56}$ A elevada tendência hemorrágica foi atribuída à elevada vascularização do tecido decidual, associada à sua friabilidade..$^{5,34,50}$

Também são relatados quadros álgicos de predomínio abdominal, enquadrados, por vezes, na manifestação clínica de hemoperitoneu e, outras vezes, simulando outros quadros de abdómen agudo, tais como apendicite. . $4,30,44,50,52$ A decídua ectópica pode levar ao desenvolvimento de compressão e irritação mecânica dos tecidos envolventes, ${ }^{5,22,30,41,48,62}$ bem como irritação química provocada por fatores humorais libertados pela própria decídua ectópica. ${ }^{50}$ O aumento de síntese de prostaglandinas pela decídua ectópica pode estimular células musculares de outros locais como, por exemplo, a camada muscular do apêndice e originar quadros clínicos de apendicite. ${ }^{50} \mathrm{~A}$ DE pode atingir dimensões consideráveis, provocando efeitos de

Tabela 2 - Manifestações clínicas associadas à decidualização ectópica

\begin{tabular}{|c|c|c|}
\hline Manifestações clínicas & & $\mathbf{n}$ \\
\hline Alterações ginecológicas & Perda hemática genital1-3,15,16,24,25 & 48 \\
\hline \multirow[t]{11}{*}{ Alterações abdominais } & Abdómen agudo & \\
\hline & a) Hemoperitoneu & 15 \\
\hline & - Durante gravidez $z^{6,9,26,29,34,55,56}$ & 8 \\
\hline & - Intra-parto ${ }^{7}$ & 1 \\
\hline & - Pós-parto $5,7,30,41,48$ & 5 \\
\hline & - Não associado a gravidez ${ }^{4}$ & 1 \\
\hline & b) Apendicite aguda ${ }^{14,30,44,50,52}$ & 9 \\
\hline & c) Outras etiologias ${ }^{1}$ & 1 \\
\hline & Nódulos cutâneos ${ }^{61,62}$ & 4 \\
\hline & Hemorragia digestiva baixa ${ }^{6,55}$ & 2 \\
\hline & Efeito de massa ${ }^{38}$ & 1 \\
\hline Alterações do sistema respiratório & Pneumotórax ${ }^{58-60}$ & 3 \\
\hline \multirow{3}{*}{ Alterações urinárias } & Hidronefrose $e^{38,64}$ & 2 \\
\hline & Disúria ${ }^{65}$ & 1 \\
\hline & Hematúria ${ }^{64}$ & 1 \\
\hline Morte fetal ${ }^{9,55,56}$ & & 3 \\
\hline
\end{tabular}

n: número total de casos descritos no conjunto dos artigos incluídos 
massa com sintomas compressivos dos órgãos adjacentes, dor por crescimento e compressão de órgãos ou nervos, obstrução digestiva, urinária e mesmo distocia mecânica, impossibilitando o parto vaginal. ${ }^{38}$ Podem ser encontrados nódulos cutâneos indolores no períneo, no umbigo ou sob a cicatriz de cesariana. ${ }^{61,62}$

Alterações do sistema respiratório também estão descritas, incluindo pneumotórax. ${ }^{58-60}$ Entre as alterações urinárias mais frequentemente relatadas temos a hidronefrose ${ }^{38,64}$ a disúria ${ }^{65}$ e hematúria. ${ }^{64}$

Estão descritos casos de morte fetal, decorrentes de choque hipovolémico materno, em consequência de graves hemoperitoneus por rotura de vasos uterinos, onde ocorreu decidualização da serosa uterina. ${ }^{9,55,56}$ Nos casos descritos, ocorreu mortalidade neonatal por imaturidade pulmonar, originada pela necessidade de terminação da gravidez pré-termo, ${ }^{9,56}$ ou por malformações pulmonares no contexto de Trissomia 21. ${ }^{55} \mathrm{~A}$ nível de repercussão materna, a histerectomia total de necessidade foi realizada com acrescida e significativa morbilidade para estas mulheres com consequências a longo prazo.

Em termos de repercussão obstétrica, atualmente não há evidência científica robusta, e a que existe é contraditória, sobre o efeito da DE no parto pré-termo. ${ }^{7,26}$ Alguns autores descrevem que a presença de reação decidual no colo não predispõe a aborto espontâneo, ${ }^{24}$ ao contrário de outros que afirmam que existe risco aumentado de aborto e de parto pré-termo. ${ }^{26}$

\section{Macroscopia}

Estas lesões podem apresentar-se sob diversos fenótipos, quer ao exame objetivo quer como achados intraoperatórios:

a) Cavidade abdominopélvica: Quando existem alterações aparentes, estão descritas como nódulos vermelhos/ hemorrágicos, amarelos, cinzentos, castanhos ou brancos, de 0,2 a $6 \mathrm{~cm}$ de dimensão máxima, ${ }^{4,38,47,68,69} \mathrm{em}$ qualquer localização da superfície peritoneal. Estes nódulos podem ser focais ou difusos, ${ }^{20}$ por vezes sob a forma de aderências peritoneais. ${ }^{22}$

b) Colo uterino: A deciduose cervical pode apresentar-se de formas distintas, dependendo da profundidade, densidade e extensão da lesão. A sua localização preferencial é a área periorificial ${ }^{66} \mathrm{e}$, macroscopicamente, pode manifestar-se como lesões planas, polipóides ou ulceradas. ${ }^{2,3,21,24,43,66}$

c) Pele: Ocorre sob a forma de nódulos eritematosos ou da coloração da pele adjacente. ${ }^{61,62}$

d) Tórax: Encontram-se descritos como nódulos intraparenquimatosos pulmonares ${ }^{58,59}$ ou na superfície diafragmática, ${ }^{60} \mathrm{com}$ dimensão máxima de $2 \mathrm{~cm}$, podendo ser bilaterais e multifocais.

e) Intestino: Na observação por colonoscopia, surge como mucosa nodular, edemaciada e friável. ${ }^{55}$

\section{Meios complementares de diagnóstico}

No sentido de esclarecimento etiológico no contexto de manifestações clínicas ou de achados macroscópicos, os meios auxiliares de diagnóstico tornam-se elementos essenciais no diagnóstico definitivo:

\section{- Imagiologia}

A radiografia do tórax pode evidenciar lesões radiopacas, bem definidas, por vezes de aspeto miliar. ${ }^{58} \mathrm{Na}$ deciduose ovárica, a ecografia pélvica mostra espessamento do estroma com neovascularização, de natureza papilar, mimetizando os achados ultrassonográficos sugestivos de malignidade. ${ }^{40}$ Nestes casos, na ressonância magnética a lesão ovárica de deciduose apresenta caracteristicamente isointensidade com a placenta, auxiliando a diferenciação

Tabela 3 - Painel dos principais anticorpos usados na técnica de imunohistoquímica para diagnóstico diferencial $20,22,33,34,38,50,59,61,75$

\begin{tabular}{|c|c|c|c|c|}
\hline Anticorpo & Deciduose & $\begin{array}{l}\text { Mesotelioma } \\
\text { deciduóide }\end{array}$ & $\begin{array}{c}\text { Carcinoma } \\
\text { metastático }\end{array}$ & Melanoma \\
\hline Recetor estrogénio & $-/+$ & - & - & - \\
\hline Recetor progesterona & + & - & - & - \\
\hline Vimentina & + & $-/+$ & - & \\
\hline Ki-1 (CD30) & + & - & & \\
\hline Citoqueratinas AE1/AE3, 5/6, MNF 116 & - & + & + & \\
\hline Antigénio membrana epitelial (EMA) & - & + & + & \\
\hline HMBE-1 & - & + & - & - \\
\hline Calretinina & - & + & - & \\
\hline Desmina & $-/+$ & $-/+$ & & \\
\hline Actina músculo liso & $-/+$ & $-/+$ & & \\
\hline HМB 45 & - & - & & + \\
\hline Proteína S100 & - & - & & + \\
\hline CD68 & - & - & - & - \\
\hline
\end{tabular}

+: marcação positiva; -: marcação negativa. 
entre decidualização e malignidade..$^{40}$

\section{- Histologia}

Apesar dos meios de imagem poderem ser auxiliares importantes no diagnóstico diferencial com entidades malignas, nem sempre a distinção entre estas duas entidades é clara, sendo o exame histológico o gold standard para o diagnóstico definitivo. Contudo, deve ser cuidadosamente ponderada a biópsia dirigida a estas lesões e avaliado o elevado risco de hemorragia associado à biópsia versus o benefício relativo ao diagnóstico e exclusão de entidades malignas. ${ }^{20,24,33,50}$

$\mathrm{Na}$ microscopia, a reação decidual surge na subserosa ou no tecido adiposo; ${ }^{26,36,37,42,50}$ por vezes, associada a inflamação. ${ }^{24}$ A presença de mitoses, necrose, pleomorfismo e hipercromasia nuclear não são frequentes. ${ }^{22,59}$

A célula decidual caracteriza-se por ser grande, com forma poligonal ou redonda, de limites bem definidos e citoplasma basófilo, eosinófilo ou anfófilo. O núcleo é tipicamente único, central e pálido, e a maioria apresenta um padrão de cromatina finamente granular, nucléolo proeminente e aumento nuclear marcado. ${ }^{22,33,42,59} \mathrm{~A}$ vacuolização é um fenómeno frequente nestas células, proporcional à idade gestacional. ${ }^{20,47} \mathrm{O}$ estroma pode conter depósitos mixóides como resultado da rotura vacuolar. ${ }^{20}$

Perante o achado intraoperatório de lesões suspeitas e em que macroscopicamente a equipa cirúrgica não consiga excluir a hipótese diagnóstica de malignidade, estará indicado o recurso ao exame extemporâneo. No entanto, a utilização de cortes de congelação pode levar à presença de artefactos de congelação ou necrose, criando problemas de diagnóstico, pois o detalhe histológico pode ser obscurecido, limitando a acuidade diagnóstica do exame extemporâneo. ${ }^{21}$

As técnicas complementares de imunohistoquímica (Tabela 3) desempenham um papel fundamental no esclarecimento etiológico perante o achado destas lesões. Entre as características imunohistoquímicas da DE destaca-se marcação positiva com anticorpos anti-recetores da progesterona, vimentina e CD30, e marcação negativa para citoqueratinas, antigénio da membrana epitelial, HMB 45 e Proteína S100.

\section{Diagnostico diferencial}

A presença de tecido decidual heterotópico pode colocar problemas de diagnóstico ao exame objetivo, em meios complementares de imagem, ao exame macroscópico e até microscópico, podendo ser confundido com outras alterações benignas (tecido de reparação, ectropion cervical, tumores benignos ou processos infeciosos) ${ }^{70}$ ou malignas. No entanto, deciduose e tumores malignos podem coexistir. ${ }^{16}$

O seu principal diagnóstico diferencial é o mesotelioma deciduóide maligno. , $11,74^{-74}$

\section{Tratamento}

Nos casos de deciduose na gravidez, estas lesões ge- ralmente involuem espontaneamente quatro a seis semanas após o parto, quando a estimulação da progesterona cessa. Assim, e uma vez que as lesões são na maioria assintomáticas, a maior parte dos casos não requer intervenção terapêutica. ${ }^{3,22,47}$

Pelo seu elevado risco hemorrágico aquando de biópsia e excisão, deve-se optar por tratamento conservador/expectante. As intervenções mais invasivas - nomeadamente hemostase cirúrgica, citorredução cirúrgica de lesões e reconstrução de órgãos - estão restritas a sintomatologia grave, cujo benefício de abordagem diagnóstica/terapêutica supera o risco elevado de hemorragia. ${ }^{5-8,26,29,48,55,60}$

\section{Prognóstico}

A DE é uma entidade histologicamente benigna e que, de um modo geral, se comporta também de uma forma benigna. O prognóstico é bom com involução das lesões geralmente até à $4^{\mathrm{a}}$ - $6^{\mathrm{a}}$ semana de pós-parto. ${ }^{20,22} \mathrm{Em}$ casos mais graves de deciduose peritoneal, recomenda-se seguimento, sob controlo ecográfico, no primeiro e sexto mês pós-parto. ${ }^{47}$

Assim, de um modo geral, a reação decidual ectópica é fisiológica, com um excelente prognóstico e resolução espontânea. $^{27}$

\section{DISCUSSÃO E CONCLUSÃO}

Apesar de a DE se tratar de uma entidade já descrita desde o final do século XIX, a evidência científica existente é limitada. A grande maioria dos artigos é de nível de evidência 4 , sobretudo baseada na descrição de casos clínicos, existindo apenas uma revisão sistemática nesta área. Torna-se também difícil estabelecer a prevalência desta patologia devido às limitações metodológicas dos diferentes artigos disponíveis. Existem artigos que executam biópsias sistemáticas com o intuito de determinar a prevalência de $\mathrm{DE}$, no entanto a metodologia e local exato de colheita das biópsias, o número total de casos estudados, os critérios de seleção da amostragem ou a descrição das técnicas de anatomia patológica utilizadas, não são descritas em todos os artigos, o que limita quer a valorização dos resultados quer a comparação destes com outros estudos. ${ }^{15,19,20,24,25,36,37,42,43}$

Este artigo de revisão procura sensibilizar os médicos para esta entidade muitas vezes subdiagnosticada. O reconhecimento das suas características clínicas, bem como o seu elevado índice de suspeição tornam-se essenciais na conduta destas doentes, para a sua abordagem adequada e manutenção do seu perfil de bom prognóstico.

\section{CONFLITO DE INTERESSES}

Os autores declaram não ter nenhum conflito de interesses relativamente ao presente artigo.

\section{FONTES DE FINANCIAMENTO}

Não existiram fontes externas de financiamento para a realização deste artigo. 


\section{REFERÊNCIAS}

1. Taussig FJ. Ectopic decidua formation. Surg Gynecol Obstet. 1906;2:292-303.

2. Lepage F, Schramm B. Deciduosis of the uterine cervix. Gynecol Obstet. 1955:54:550-63.

3. Lapan B. Deciduosis of the cervix and vagina simulating carcinoma. Am J Obstet Gynecol. 1949;58:743-7.

4. Tang LC, Cheung MY, Ma HK. Intraperitoneal bleeding from ectopic decidua following hormonal contraception. Case report. $\mathrm{Br} \mathrm{J}$ Obstet Gynaecol. 1985;92:102-3.

5. O'Leary SM. Ectopic decidualization causing massive postpartum intraperitoneal hemorrhage. Obstet Gynecol. 2006;108:776-9.

6. Nishikawa A, Kondoh E, Hamanishi J, Yamaguchi K, Ueda A, Sato $Y$, et al. lleal perforation and massive intestinal haemorrhage from endometriosis in pregnancy: case report and literature review. Eur J Obstet Gynecol Reprod Biol. 2013;170:20-4.

7. Park J, Thomas P, Staecker D. Hemorrhage of ectopic deciduosis necessitating emergent surgical resection. Kansas J Med. 2011:11-3

8. Dharan M. Hyaline globules in ectopic decidua in a pregnant woman with cervical squamous cell carcinoma. Diagn Cytopathol. 2009;37:696-

9. Aziz U, Kulkarni A, Lazic D, Cullimore JE. Spontaneous rupture of the uterine vessels in pregnancy. Obstet Gynecol. 2004;103:1089-91.

10. Howick J. Oxford Centre for Evidence-based Medicine - Levels of Evidence March 2009. [Consultado 2014 set 10]. Disponível em: http://www.cebm.net/oxford-centre-evidence-based-medicine-levelsevidence-march-2009/.

11. Canlorbe G, Goubin-Versini I, Azria E, Abdul-Razak R, Muray JM. Ectopic decidua: variability of presentation in pregnancy and differential diagnoses. Gynecol Obstet Fertil. 2012;40:235-40.

12. Weller $\mathrm{CV}$. The ectopic decidual reaction and its significance in endometriosis. Am J Pathol. 1935;11:287-90

13. Ober WB, Grady HG, Schoenbucher AK. Ectopic ovarian decidua without pregnancy. Am J Pathol. 1957;33:199-217.

14. Reis RA, Sinykin MB. Ectopic decidua in the vermiform appendix. Am J Obstet Gynecol. 1940;39:870-2.

15. Orr CJ, Pedlow PR. Deciduosis of the cervix manifesting as antepartum hemorrhage and simulating carcinoma. Am J Obstet Gynecol. 1961;82:884-6

16. Cobb CJ. Ectopic decidua and metastatic squamous carcinoma: presentation in a single pelvic lymph node. J Surg Oncol. 1988;38:1269.

17. Wu DC, Hirschowitz S, Natarajan S. Ectopic decidua of pelvic lymph nodes: a potential diagnostic pitfall. Arch Pathol Lab Med. 2005;129:e117-20.

18. Masouridou S, Mamopoulos A, Mavromatidis G, Karagiannis V. Endometriosis and perinatal outcome - a systematic review of the literature. Curr Women's Health Rev. 2012;8:121-30.

19. Zaytsev P, Taxy JB. Pregnancy associated ectopic decidua. Am J Surg Pathol. 1987;11:526-30.

20. Buttner A, Bassler R, Theele C. Pregnancy-associated ectopic decidua (deciduosis) of the greater omentum. An analysis of 60 biopsies with cases of fibrosing deciduosis and leiomyomatosis peritonealis disseminata. Pathol Res Pract. 1993;189:352-9.

21. Gornall AS, Naftalin NJ, Brown LJ, Konje JC. Massive necrosis of cervical ectopic decidua presenting in labour. $\mathrm{Br} \mathrm{J}$ Obstet Gynaecol. 2000;107:573-5

22. Bolat F, Canpolat T, Tarim E. Pregnancy-related peritoneal ectopic decidua (deciduosis): morphological and clinical evaluation. Turk Patoloji Derg. 2012;28:56-60.

23. Ellis CL, Maleki Z, Ali SZ. Ectopic decidua in abdominal washings found intraoperatively at cesarean section. Diagn Cytopathol. 2010;38:740-1.

24. McGee WB, Slate TA. Decidual reaction of the cervix - a review of 27 cases. Calif Med. 1955;82:306-8.

25. Le Coz A, Mazerolles J, Masson M.A propos de huit cas de deciduose du col uterin. Rev Fr Gynecol Obstet. 1967;62:535-9.

26. Semenova T, Metello J, Almeida M, Leite C, Santos A. Decidualização ectópica como causa de hemorragia intra-cesariana. Acta Obstet Ginecol Port. 2013;7:316-9.

27. Abramowicz S, Kouteich K, Gremain J, Sabourin JC, Marpeau L, Sergent F. Giant ectopic peritoneal and omental deciduosis mimicking a peritoneal carcinomatosis. Gynecol Obstet Fertil. 2014;42:182-4.

28. Burnett RA, Millan D. Decidual change in pelvic lymph nodes: a source of possible diagnostic error. Histopathology. 1986;10:1089-92.

29. Melody GF. Deciduation and massive hemorrhage of the omentum in the final month of pregnancy. West J Surg Obstet Gynecol. 1950;58:460-2.

30. Hulme-Moir I, Ross MS. A case of early postpartum abdominal pain due to haemorrhagic deciduosis peritonei. J Obstet Gynaecol $\mathrm{Br}$ Commonw. 1969;76:746-9.

31. Kondi-Pafiti A, Grapsa D, Kontogianni-Katsarou K, Papadias K, KairiVassilatou E. Ectopic decidua mimicking metastatic lesions-repor of three cases and review of the literature. Eur J Gynaecol Oncol. 2005;26:459-61.

32. Shukla S, Pujani M, Singh SK. Ectopic decidual reaction mimicking peritoneal tubercles: a report of three cases. Indian J Pathol Microbiol. 2008:51:519-20

33. Acuda C, Wise O, Chaudhri P. Diffuse deciduosis occurring within an ovarian cystadenoma - a clinical-pathological mimic of a malignant process. Diagn Histopathol. 2011;17:313-6

34. Kondoh E, Shimizu M, Kakui K, Mikami Y, Tatsumi K, Konishi I. Deciduosis can cause remarkable leukocytosis and obscure abdominal pain. J Obstet Gynaecol Res. 2012;38:1376-8.

35. Galao AO, Ramos-Lima LF, Schuh F, Golbspan L, Grezzana TJ. Ectopic decidua in pregnancy discovered incidentally during caesarean delivery. J Obstet Gynaecol. 2013;33:207-8.

36. Israel SL, Rubenstone A, Meranze DR. The ovary at term. I. Decidua-like reaction and surface cell proliferation. Obstet Gynecol. 1954;3:399-407.

37. Herr JC, Heidger PM Jr., Scott JR, Anderson JW, Curet LB, Mossman HW. Decidual cells in the human ovary at term. I. Incidence, gross anatomy and ultrastructural features of merocrine secretion. Am J Anat. 1978;152:7-27.

38. Malpica A, Deavers MT, Shahab I. Gross deciduosis peritonei obstructing labor: a case report and review of the literature. Int J Gynecol Pathol. 2002;21:273-5.

39. Kinra $P$, Sen A, Sharma J. Ectopic decidual reaction: a case report MJAFI. 2006:62:280-1.

40. Machida S, Matsubara S, Ohwada M, Ogoyama M, Kuwata T, Watanabe $\mathrm{T}$, et al. Decidualization of ovarian endometriosis during pregnancy mimicking malignancy: report of three cases with a literature review. Gynecol Obstet Invest. 2008;66:241-7.

41. Boztosun A, Sumer D, Cetin M, Cetin A. Idiopathic spontaneous hemoperitoneum during early postpartum period: Case report. Turk Klin Tip Bilim Derg. 2012;32:1718-20.

42. Schneider V, Barnes LA. Ectopic decidual reaction of the uterine cervix frequency and cytologic presentation. Acta Cytol. 1981;25:616-22.

43. Chapman GW, Savage EW, Salem FA. Cervical deciduosis and intraepithelial neoplasia. J Natl Med Assoc. 1979;71:787-9.

44. Dogan E, Okyay E, Saatli B, Olgan S, Sarioglu S, Koyuncuoglu M. Tuba ovarian abscesses formation from decidualized ovarian endometrioma after appendiceal endometriosis presenting as acute appendicitis in pregnancy. Iran J Reprod Med. 2012;10:275-8.

45. Adhikari LJ, Shen R. Florid diffuse peritoneal deciduosis mimicking carcinomatosis in a primigravida patient: a case report and review of the literature. Int J Clin Exp Pathol. 2013;6:2615-9.

46. Salehgargari S, Sahebdel B, Zare A, Abolhassani H. Ectopic decidual reaction mimicking irritable bowel syndrome: a case report. Acta Med Iran. 2014;52:88-90.

47. Cruz DB, Dhamer T, da Rocha VW, Dupont RF. Diffuse peritoneal deciduosis mimicking metastatic lesions. BMJ Case Rep. 2014

48. Richter MA, Choudhry A, Barton JJ, Merrick RE. Bleeding ectopic decidua as a cause of intraabdominal hemorrhage. A case report. $J$ Reprod Med. 1983;28:430-2.

49. Sanes S, Liber AF. Ectopic decidua in vermiform appendix - Showing (1) acute appendicitis and (2) acute periappendicitis. Am J Obstet Gynecol. 1943;46:719-21.

50. Suster S, Moran CA. Deciduosis of the appendix. Am J Gastroenterol. 1990:85:841-5

51. Rodriguez FJ, Abraham SC, Sendelbach KM, Nascimento AG. Florid decidual reaction mimicking gastrointestinal malignancy in a primipara woman. Histopathology. 2006;49:82-5

52. Lesaffer J, Feryn T, Proot L. Pregnancy-associated ectopic decidua of the appendix. Acta Chir Belg. 2009;109:93-4

53. Covell LM, Disciullo AJ, Knapp RC. Decidual change in pelvic lymph nodes in the presence of cervical squamous cell carcinoma during pregnancy. Am J Obstet Gynecol. 1977;127:674-6.

54. Mills SE. Decidua and squamous metaplasia in abdominopelvic lymph nodes. Int J Gynecol Pathol. 1983;2:209-15.

55. Bashir RM, Montgomery EA, Gupta PK, Nauta RM, Crockett SA, Collea $\mathrm{JV}$, et al. Massive gastrointestinal hemorrhage during pregnancy caused 
by ectopic decidua of the terminal ileum and colon. Am J Gastroenterol. 1995;90:1325-7.

56. Mizumoto Y, Furuya K, Kikuchi Y, Aida S, Hyakutake K, Tamai S, et al. Spontaneous rupture of the uterine vessels in a pregnancy complicated by endometriosis. Acta Obstet Gynecol Scand. 1996;75:860-2.

57. Kularbkaew C, Yutanawiboonchai W, Pairojkul C. Molar pregnancy associated ectopic decidua: report of a case and review of the literature. J Med Assoc Thai. 1998;81:918-22.

58. Courtin P, Sepulveda S, Sauvageon X, Sfoggia D, Prudent J, Girard C, et al. Suspicion of pulmonary endometriosis in a woman presenting with pulmonary deciduosis. Apropos of a case. Cah Anesthesiol. 1991;39:435.

59. Flieder DB, Moran CA, Travis WD, Koss MN, Mark EJ. Pleuro-pulmonary endometriosis and pulmonary ectopic deciduosis: a clinicopathologic and immunohistochemical study of 10 cases with emphasis on diagnostic pitfalls. Hum Pathol. 1998;29:1495-503.

60. Kim YD, Min KO, Moon SW. Thoracoscopic treatment of recurrent pneumothorax in a pregnant woman: a case of ectopic deciduosis. Thorac Cardiovasc Surg. 2010;58:429-30.

61. Fair KP, Patterson JW, Murphy RJ, Rudd RJ. Cutaneous deciduosis. J Am Acad Dermatol. 2000;43:102-7.

62. Natale KE, Royer MC, Rush WL, Lupton GP. Cutaneous deciduosis: a report of two cases of an unusual pseudomalignancy. J Cutan Pathol. 2012;39:777-80.

63. Agarwal J, Gupta JK. Ectopic decidua in association with adenomyosis presenting as fibroids in pregnancy-a case report. Indian J Pathol Microbiol. 1997;40:91-3.

64. Bettinger HF. Ectopic decidua in the renal pelvis. J Pathol Bacteriol. 1947;59:686.

65. Szopinski TR, Sudol-Szopinska I, Dzik T, Borowka A. Ectopic decidual reaction in the urinary bladder presenting as a vesical tumor. Urology. 2009;74:1232-3.

66. Gilardi EM, Migliarini AM, Remotti G, Ferrario I, Stillitani M. Cervical deciduosis: What clinical relevance? Cervix and the Lower Female Genital Tract. 1988;6:303-9.

67. Clement PB. Tumor-like lesions of the ovary associated with pregnancy. Int J Gynecol Pathol. 1993;12:108-15.

68. Osullivan D, Heffernan CK. Deciduosis peritonei in pregnancy - report of 2 cases respectively simulating carcinoma and tuberculosis. J Obstet Gynaecol. 1960;67:1013-6.

69. Kwan D, Pang LS. Deciduosis peritonei. J Obstet Gynaecol Br Commonw. 1964;71:804-6.

70. Bret J, Coupez F, De Brux J. Deciduosis of the uterine cervix: clinical \& colposcopic aspects. Gynecol Obstet. 1959;58:199-207.

71. Reis-Filho JS, Pope LZ, Milanezi F, Balderrama CM, Serapiao MJ, Schmitt FC. Primary epithelial malignant mesothelioma of the pericardium with deciduoid features: cytohistologic and immunohistochemical study. Diagn Cytopathol. 2002;26:117-22.

72. Serio G, Scattone A, Pennella A, Giardina C, Musti M, Valente T, et al. Malignant deciduoid mesothelioma of the pleura: report of two cases with long survival. Histopathology. 2002;40:348-52.

73. Mourra N, de Chaisemartin C, Goubin-Versini I, Parc R, Flejou JF. Malignant deciduoid mesothelioma: a diagnostic challenge. Arch Pathol Lab Med. 2005;129:403-6.

74. Nascimento AG, Keeney GL, Fletcher CD. Deciduoid peritoneal mesothelioma. An unusual phenotype affecting young females. Am J Surg Pathol. 1994;18:439-45.

75. Ustun H, Astarci HM, Sungu N, Ozdemir A, Ekinci C. Primary malignant deciduoid peritoneal mesothelioma: a report of the cytohistological and immunohistochemical appearances. Diagn Cytopathol. 2011;39:402-8. 